\title{
Comparison of the macroscopic properties of field-accelerated electrons in dry air and in pure oxygen
}

\author{
G. Fournier, J. Bonnet and D. Pigache \\ ONERA, 92320 Châtillon, France
}

(Reçu le 7 décembre 1979, accepté le 15 février 1980)

\begin{abstract}
Résumé. - La résolution numérique de l'équation de Boltzmann pour un gaz ionisé donne les propriétés macroscopiques des électrons soumis à l'action d'un champ électrique dans l'air sec et dans l'oxygène pur. En vue de la synthèse de l'ozone, le rendement de dissociation de l'oxygène est toujours d'autant plus grand que le champ est élevé. Comparée au cas de l'oxygène pur, sa dissociation dans l'air s'obtient beaucoup moins facilement que ne le laisse prévoir sa concentration.
\end{abstract}

\begin{abstract}
The numerical solution of the Boltzmann equation for an ionized gas yields the macroscopic properties of electrons accelerated by an electric field in dry air and in pure oxygen. For the purpose of ozone generation, the stronger the field, the better the efficiency of oxygen dissociation. In air, the oxygen dissociation is found to be much less easy than that at the same amount of pure oxygen.
\end{abstract}

The macroscopic properties of the electrons in a given gas (pure gas or gas mixture) are often functions of the only variable $E / N$ (electric field/gas density). The concentrations of metastables or ionized species may sometimes be additional variables but, for the present cases, they are assumed to be low enough to be neglected. The last two variables, to be taken into account in some cases, are the neutral temperature $T$ and the gas density $N$; the following computations are performed under standard conditions $(T=273 \mathrm{~K}$ and $N=2.69 \times 10^{19} \mathrm{~cm}^{-3}$ ). Nevertheless, for the gases and the range of $E / N$ to be considered in this paper, $T$ may be varied over a large range without any effect on the results; $N$ changes only the three-body attachment coefficient, which is proportional to it, but it has no effect on the other macroscopic properties for values lower or a little higher than the standard one.

The more usual macroscopic properties are the drift velocity $\left(v_{\mathrm{d}}\right)$, the electron temperature $\left(T_{\mathrm{e}}=\right.$ average energy $\times 2 / 3$ ), the ratio of the diffusion coefficient over the mobility $(D / \mu)$, the reaction rates and the energy branching ratios for the various elastic and inelastic processes taken in to account. These properties are computed from the electron distribution function which is itself a solution of the classical Boltzmann equation for an ionized gas. The computer code which is used is based upon previous work [1].

Although literature deals with many gases (mainly pure gases and laser mixtures), air has only been considered in the preliminary work by Hake and
Phelps [2], which is based upon obsolete assumptions and does not provide the main macroscopic properties. The reason for this is related to the difficulty to have a satisfactory and coherent set of data available for oxygen cross-sections. Some differences between the data used in a recent paper on pure oxygen by Masěk et al. [3] and those used here $[4,5]$ are an illustration of the problem. The in terest in air is not only due to the fact that it is the ambient medium; it is also a raw material for the generation of ozone in an electric discharge for the purpose of water purification. Any improvement of present industrial systems requires an insight in ozone synthesis processes, among which the most important is the dissociation of molecular oxygen by electrons. The following results allow a comparison of the possibilities for converting electric energy in to oxygen dissociation in dry air and in pure oxygen. In addition, this investigation is of direct interest for the understanding of atmospheric electricity phenomena.

Elastic collisions with $\mathrm{N}_{2}, \mathrm{O}_{2}, \mathrm{Ar}$ and $\mathrm{CO}_{2}$ are taken into account. The inelastic processes taken into account for $\mathrm{N}_{2}$ and $\mathrm{O}_{2}$ are summarized in table $\mathrm{I}$. Inelastic processes with $\mathrm{Ar}$ and $\mathrm{CO}_{2}$ are not given in detail since their effect is quite minor. Dry air composition is $\left[\mathrm{N}_{2}: \mathrm{O}_{2}: \mathrm{Ar}: \mathrm{CO}_{2}=7808: 2095: 93: 3\right]$.

The differences between dry air and pure oxygen are significant for all the results displayed. Figure 1 gives the variations of the macroscopic parameters $v_{\mathrm{d}}, T_{\mathrm{e}}$ and $D / \mu$ versus $E / N\left(1 \mathrm{Td}=10^{-17} \mathrm{~V} . \mathrm{cm}^{2}\right)$. The last 
Table I. - Inelastic processes taken into account with their energy loss in $\mathrm{eV}$.

\section{Oxygen [4]$$
\text { ygen }[4]
$$

$0.051-3$ body attachmen $t$

0.007 -rotations

$0.19,0.38,0.57,0.75$-vibrations

0.977-a ${ }^{1} \Delta_{\mathrm{g}}$ excitation

1.627-b ${ }^{1} \Sigma_{\mathrm{g}}^{+}$excitation

4.4-dissociative attachment

4.5-electronic excitation(s)

6-dissociative ? electronic

excitation(s)

8.4-B ${ }^{3} \Sigma_{\mathrm{u}}{ }^{-}$dissociative excitation

10 -electronic excitation

12.2-ionization

14.7-electronic excitation

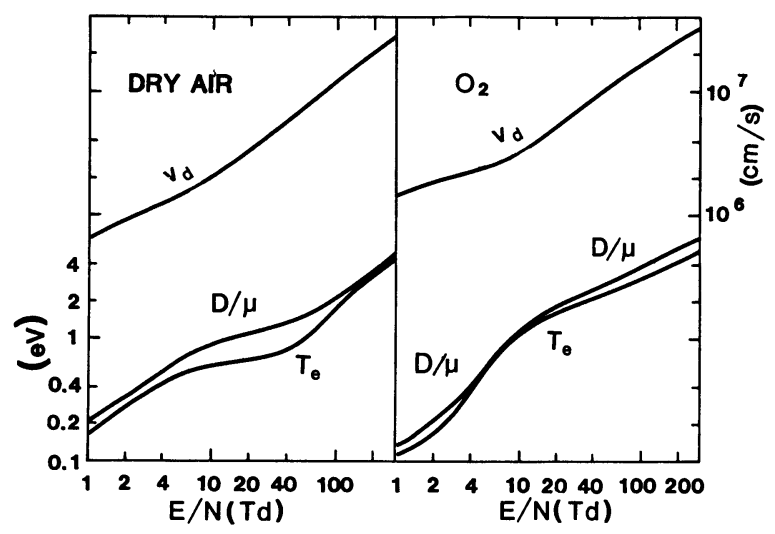

Fig. 1. - Drift velocity and characteristic energy variations versus reduced electric field.

two are equal if the distribution function is Maxwellian; the difference between them results from the shift of the actual distribution with respect to the Maxwellian one. Figure 2 gives reaction rates as $\alpha / N_{i}$; (the rate coefficients in $\mathrm{cm}^{3} / \mathrm{s}$ are the products of $\alpha / N_{i}$ in $\mathrm{cm}^{2}$, times the drift velocity in $\mathrm{cm} / \mathrm{s}$ ). Figure 3 gives the energy branching ratios for some groups of processes.

The differences of the present results for pure oxygen with those by Masěk et al. [3] are minor.

For air, it is clear that energy transfers to $\mathrm{Ar}$, to $\mathrm{CO}_{2}$ and by elastic collisions are all negligible. At lower fields (a few Td), the largest fraction of the energy is transferred to oxygen vibrations. At moderate fields (a few tens of $\mathrm{Td}$ ) almost all the energy is transferred to nitrogen vibrations; this is made at the expense of oxygen singlets which are never pumped as efficiently in air as they are in the pure gas. At higher fields, the electronic excitations of oxygen (some of

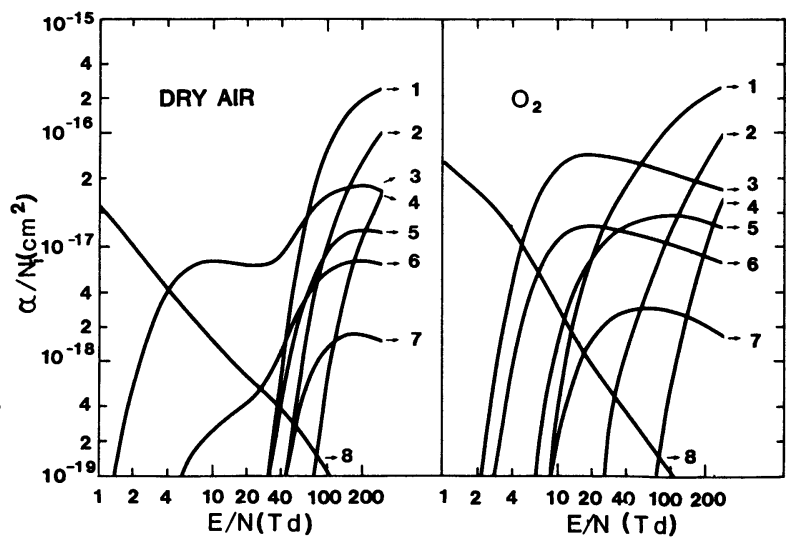

Fig. 2. - Variations of some reaction rates versus reduced electric field. $1=6 \mathrm{eV} ; 2=8.4 \mathrm{eV} ; 3=\mathrm{a}^{1} \Delta_{\mathrm{g}} ; 4=$ ionization $; 5=4.5 \mathrm{eV}$; $6=\mathrm{b}^{1} \sum_{\mathrm{g}}{ }^{+} ; 7=$ dissociative attachment $; 8=3$-body attachment.

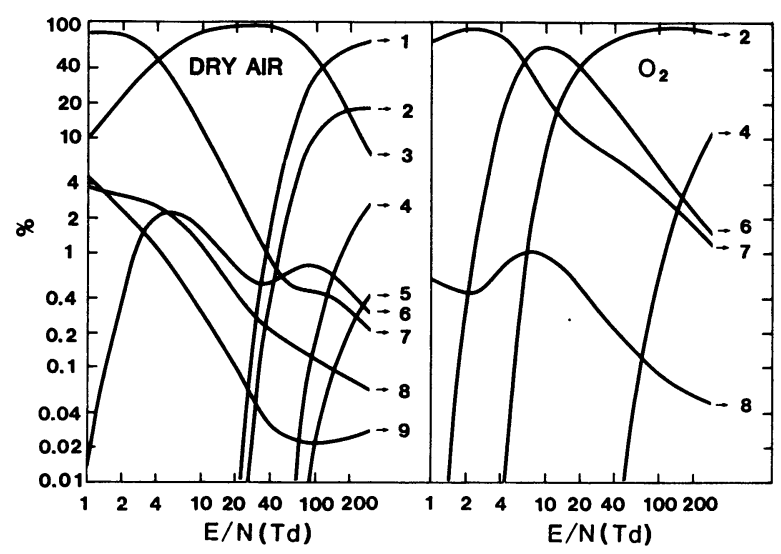

Fig. 3. - Variations of energy branching ratios versus reduced electric field. $1=\mathrm{N}_{2}$, except vibrations; $2=\mathrm{O}_{2},>4 \mathrm{eV}$ except ionization ; $3=\mathrm{N}_{2}$, vibration s; $4=\mathrm{O}_{2}$, ionization ; $5=\mathrm{Ar} ; 6=\mathrm{O}_{2}$, singlets $; 7=\mathrm{O}_{2}$, vibrations $; 8=$ elastic $; 9=\mathrm{CO}_{2}$.

which can be dissocia tive) receive an energy fraction in agreement with the $\mathrm{O}_{2}$ fraction in air. It should be noted that the $\mathrm{N}_{2}$ excitations for this range of field are related mainly to the $\mathrm{A}^{3} \Sigma_{\mathrm{u}}^{+}$metastable and that, consequently, a fraction of their energy can go back to electrons via superelastic collisions.

As a conclusion, it is possible to assess clearly that the larger fields yield the better dissociation efficiency for the purpose of ozone generation. However, with respect to the case of pure oxygen, $\mathrm{O}_{2}$ dissociation in air is much less easy to achieve than predicted from its concentration since the proper range is then closer to the breakdown field (Figs. 2 and 3) : the breakdown field is close to that for which the curves of ionization and dissociative attachment cross each other on figure 2 .

\section{References}

[1] Bonnet, J., Dahan, C., Fournier, G. and Pigache, D., Calcul des paramètres macroscopiques et des fonctions de distribution électroniques dans les plasmas de $\mathrm{CO}_{2}-\mathrm{N}_{2}-\mathrm{He}$. Note Technique ONERA no $238,1974$.

[2] Hake, R. D. and Phelps, A. V., Phys. Rev. 158 (1967) 70-84.

[3] MasěK, K., LÁsKa, L. and RŮžıČKa, T., Czech. J. Phys. B 28 (1978) 1321-1334.
[4] Lawton, S. A. and Phelps, A. V., J. Chem. Phys. 69 (1978) 1055-1068, with additional data of $11 / 3 / 1977$ from A. V. PHeLPS, private communication.

[5] KIEFFER, L. J., A compilation of electron collision cross-section data for modeling gas discharge lasers, JILA Information Center Report 13, Boulder, Colorado, USA, 1973 , with author's corrections for $N_{2}$ vibrations. 